\title{
Prevalence of congenital heart defects and persistent pulmonary hypertension of the neonate with Down syndrome
}

\author{
Michel Emile Weijerman • A. Marceline van Furth • Maurike D. van der Mooren • \\ Miriam M. van Weissenbruch • Lukas Rammeloo • Chantal J. M. Broers • \\ Reinoud J. B. J. Gemke
}

Received: 12 February 2010 / Accepted: 6 April 2010 /Published online: 23 April 2010

(C) The Author(s) 2010. This article is published with open access at Springerlink.com

\begin{abstract}
The aim of this study was to assess the prevalence of congenital heart defects (CHDs) and persistent pulmonary hypertension of the neonate (PPHN) in children with Down syndrome (DS) and to assess its impact on neonatal factors. It was a prospective study of a birth cohort of children with DS born between 2003 and 2006 registered by the Dutch Paediatric Surveillance Unit (DPSU). A CHD occurred in $43 \%$ of 482 children with trisomy 21. Atrioventricular septal defect was found in $54 \%$, ventricular septal defect in $33.3 \%$ and patent ductus arteriosus in $5.8 \%$. The incidence of PPHN in DS was $5.2 \%$, which is significantly higher than the general population $(p<0.001)$. The reported mortality in newborns with DS was overall $3.3 \%$ and was still significant higher in children with a CHD versus no CHD (5.8\% versus $1.5 \%)$
\end{abstract}

M. E. Weijerman · A. M. van Furth · M. D. van der Mooren •

C. J. M. Broers · R. J. B. J. Gemke

Department of Paediatrics,

Subdivision of General Paediatrics and Infectious Diseases,

VU University Medical Center,

Amsterdam, the Netherlands

M. M. van Weissenbruch

Department of Paediatrics, Subdivision of Neonatology,

VU University Medical Center,

Amsterdam, the Netherlands

\section{Rammeloo}

Department of Paediatrics, Subdivision of Paediatric Cardiology,

VU University Medical Center,

Amsterdam, the Netherlands

\section{E. Weijerman $(\bowtie)$}

Department of Paediatrics and Infectious Diseases,

VU University Medical Center,

P.O. Box 7057, 1007 MB Amsterdam, the Netherlands

e-mail: weijerman@vumc.nl
( $p=0.008$ ). The presence of CHD in children with DS had no influence on their birth weight, mean gestational age and Apgar score. In neonates with DS, we found not only a $43 \%$ prevalence of CHD, but also a high incidence of PPHN at $5.2 \%$. Early recognition of the cardiac condition of neonates with DS seems justified.

Keywords Down syndrome $\cdot$ Prevalence $\cdot$ Persistent pulmonary hypertension of the neonate $\cdot$ Congenital heart defects $\cdot$ Neonate

\section{Introduction}

Down syndrome (DS) is the most common autosomal chromosome anomaly, affecting 16 out of every 10,000 live births in the Netherlands [19]. DS is frequently associated with congenital heart defects (CHD). Indeed CHDs are considered to be the most important clinical phenomenon of DS highly relevant to morbidity and decisive in infant mortality [16]. Over recent decades, there has been a substantial increase in the life expectancy of children with DS in general with an improvement in average life expectancy from 12 years in the 1940 s to 60 years nowadays [16]. This increase in life expectancyhas mainly been due to the successful early surgical treatment of CHD in children with DS $[5,9,10,16]$.

Atrio-ventricular septal defect (AVSD), ventricular septal defect (VSD) and atrial septal defect (ASD) are the most common forms of CHD and recent reports have shown that the distribution of CHDs in children with DS may vary with ethnicity $[3,4,7,8,14,15,17,20]$.

In addition, Shah et al. have recently identified persistent pulmonary hypertension of the neonate (PPHN) in 24 out of 175 children with DS retrospectively; this 
increased prevalence in children with DS has not previously been identified as a specific problem among children with DS [13].

In the present study, we describe the prevalence of CHDs in neonates with DS in the Netherlands, determine the incidence of PPHN in this population and analyse the effect of a CHD on other neonatal factors.

\section{Patients and methods}

Our study includes all live births of children born between January 1, 2003 and December 31, 2006 who were diagnosed with DS and were reported to the Dutch Paediatric Surveillance Unit (DPSU). The DPSU is a national registry used by paediatricians to report various paediatric disorders, including DS. For each DS case reported to the DPSU, a questionnaire was sent to the attending pediatrician. The completed forms were returned to the Down Syndrome Study Group at the VU University Medical Center, Amsterdam. The method and details of the data collection have already been described earlier, and in 2003 the response rate for the DPSU was $96 \%$ and the overall response rate between 2003 and 2006 was 93.3\% [19].

The diagnosis of DS was made in $91 \%$ in the first 7 days of life in the Netherlands, and most questionnaires were returned within the first 3 months after birth. In the Netherlands, $20 \%$ of the population under the age of 18 is from an ethnic minority background [19].

Only children with a karyotype analysis (antenatal or postnatal) were included. To ensure the homogeneity of the children with DS, all children with DS due to mosaicism or translocation were excluded. Children with DS for whom information regarding their cardiac status was absent or incomplete were also excluded. The Dutch DS screening program carries out an echocardiogram within the first 3 months of birth. We used the following items in the questionnaire for the analyses: sex, chromosomal analysis, ethnicity, cardiac evaluation, birth weight, gestational age of birth, 5-min Apgar score and the presence of PPHN. The diagnosis of PPHN was based on a clinical assessment and echocardiogram in the first week of life performed by neonatologists or paediatric cardiologists who answered the questionnaires. PPHN is defined, if the pulmonary pressure exceeds the systemic pressure, as being present a right-to-left shunt and is diagnosed by echocardiography. The use of a high percentage of oxygen is needed usually without a reaction on $100 \%$ oxygen and a difference in oxygen saturation measured pre-ductal and post-ductal, when a PDA is present $[1,13,18]$. Secundum ASD and persistent foramen ovale (PFO) were excluded as a CHD.
Statistical analysis

The binomial test was used to compare the prevalence of CHD and PPHN in children with DS and the Dutch reference population, respectively. We tabulated the frequency of CHD and the combinations of CHDs. Statistical comparisons of birth weight and gestational age for children with DS with and without CHD were made with independent sample $t$ tests. Because of the influence of the gestational age on birth weight, we corrected birth weight for gestational age by using a multiple regression model. We used the Mann-Whitney test to compare nonparametric variables (e.g. Apgar score) and the Chi-square test for paired comparison of properties (e.g. asphyxia, ethnicity and mortality). Statistical analysis was performed with SPSS 16.0 statistical software. A $p$ value of $<0.05$ was considered significant.

\section{Results}

Study group

The DPSU registered 820 children with DS who were born between January 1, 2003 and December 31, 2006. A total of 630 questionnaires were returned by the DPSU, and of these, 482 included complete data regarding the cardiac status. A CHD was reported in $207(43 \%)$ of the children with trisomy 21 , which is significantly higher than the prevalence of CHD in the Dutch reference population $(0.62 \%, p<0.001)[12]$ (Fig. 1).

In 2003, the prevalence in the Netherlands of live and still-born children with DS was 26.8 per 10,000 pregnancies compared with 16 newborns with DS per 10,000 live births; there were no exact data on the prevalence of CHD in these stillborn children with DS [19].

\section{Congenital heart defects}

The data for CHD in children with DS are summarized in Table 1. In 2.4\% $(n=5)$ of children, the CHD did not match any of the categories mentioned and thus these were thus counted as 'other'. In $28.9 \%(n=60)$ of the children, there were multiple cardiac defects (i.e. a combination of defects).

\section{Ethnicity}

The ethnic background of children with DS was reported for 404 children and was predominantly Caucasian (77.5\%, $n=313)$. Of these Caucasian children, $127(40.6 \%)$ had a CHD. In $22.5 \%(n=91)$, one or both of the parents came from an ethnic minority group most of which were from a 


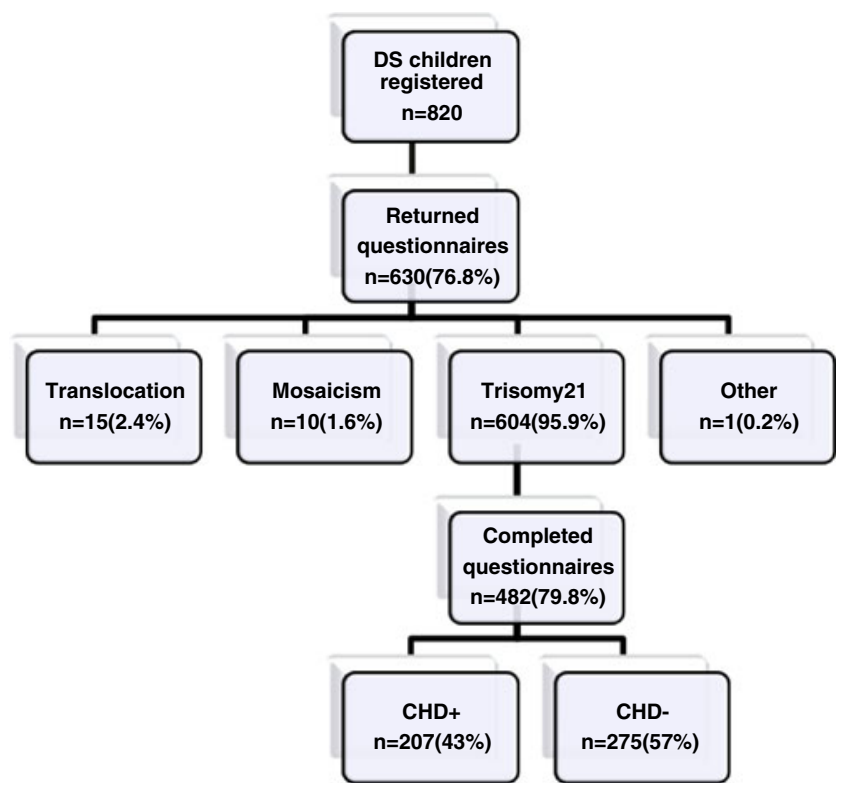

Fig. 1 Children with Down syndrome (DS) in the Netherlands born in the period January 1, 2003 to December 31, 2006 with and without congenital heart defect (CHD). Asterisk (*) one child had a combination of trisomy 21 and triple $\mathrm{X}(0.2 \%)$

Moroccan, Turkish or Antillean background. Within this ethnic group, $42.9 \%(n=39)$ of the children with DS had a CHD. There were no differences in the distribution of CHDs neither in the ethnic group compared to the Caucasian group nor within the ethnic subgroups. The data on ethnicity were missing for 78 children with DS.

Persistent pulmonary hypertension of the neonate

PPHN was reported in 25 children with DS; an incidence of $5.2 \%$, which is significant, elevated in comparison with the reported $0.1 \%$ in the general population $(p<0.001)$ [18]. Table 2 shows the distribution of CHDs in children with DS and PPHN of which $36 \%(n=9)$ of the children had no CHD.

\section{Neonatal variables}

The presence or absence of a CHD had no significant relationship with birth weight and gestational age. The mean birth weights of the children with and without a CHD were 2,926 and $3,013 \mathrm{~g}$, respectively $(95 \% \mathrm{CI} ;-270-87)$, and the mean gestational age of the children with DS with and without CHD were 38.0 and 38.3 weeks, respectively ( $95 \% \mathrm{CI} ;-0.64-0.16)$. The median of the Apgar score at 5 min was nine in children with DS both with and without a CHD. Also, the frequency of asphyxia (Apgar score $<6$ ) in children with DS with a CHD did not differ from those without a CHD (1.6\% versus $2.8 \%)$ $(p=0.39)$.
Table 1 Children with Down syndrome and a congenital heart defect (CHD; total $n=207$ )

\begin{tabular}{lrr}
\hline Congenital heart defect (CHD) & $N$ & Percent \\
\hline Single defect & 147 & 71.0 \\
AVSD & 92 & 44.4 \\
VSD & 38 & 18.4 \\
PDA & 12 & 5.8 \\
Other & & \\
Hypoplastic pulmonary arteries & 1 & 0.5 \\
COA & 1 & 0.5 \\
PS & 2 & 1.0 \\
AR & 1 & 0.5 \\
Combination of defects & 60 & 29.0 \\
AVSD + TOF & 3 & 1.5 \\
AVSD + ASD & 6 & 2.9 \\
AVSD + other & & \\
Hypoplastic aortic arch & 1 & 0.5 \\
IAA-b & 1 & 0.5 \\
PS & 1 & 0.5 \\
TOF & 8 & 3.9 \\
VSD + ASD & 29 & 14.0 \\
VSD + overriding AO & 2 & 1.0 \\
ASD + PS & 7 & 3.4 \\
Other & & \\
CAT-II + IAA-a & 1 & 0.5 \\
DAA & & \\
Total & & \\
\hline
\end{tabular}

$A S D$ secundum atrial septal defect, $A V S D$ atrioventricular septal defect, $V S D$ ventricular septal defect, $P D A$ patent ductus arteriosus, TOF Tetralogy of Fallot, IAA-a interruption of the aortic arch type a, $I A A-b$ interruption of the aortic arch type b, COA coarctation of the aorta, $P S$ pulmonary valve stenosis, $A R$ aortic regurgitation, CAT-II common arterial trunk type II, $D A A$ double aortic arch

\section{Mortality}

The reported mortality among the neonates with DS was 16 out of $482(3.3 \%)$. Most of the 16 children with DS died within 30 days of birth $(n=11)$, one died on day 39 and

Table 2 Children with Down syndrome and persistent pulmonary hypertension of the neonate (PPHN) $(n=25)$ and the distribution of a concomitant congenital heart defect (CHD)

\begin{tabular}{lcc}
\hline Congenital heart defect (CHD)a & $N$ & Percent \\
\hline AVSD & 7 & 28 \\
VSD & 6 & 24 \\
VSD + ASD & 3 & 12 \\
No CHD & 9 & 36 \\
Total & 25 & 100 \\
\hline
\end{tabular}

$A S D$ atrial septal defect, $A V S D$ atrioventricular septal defect, VSD ventricular septal defect 
another on day 318 following birth. The data of the remaining three non-survivors are missing. Among the neonates with DS who died, 12 children had a CHD (12 out of 207 CHD children (5.8\%)) and four children had no CHD (4 out of 275 non CHD children, 1.5\%). This was a significant difference in mortality among the children with DS with and without a CHD, $(p=0.008)$. The causes of death in the 12 children with DS and a CHD were as follows: respiratory distress ( $n=3$, all AVSD), neonatal infection $(n=2$, AVSD and aortic regurgitation), asphyxia ( $n=1$, AVSD), post cardiac operation ( $n=1, \mathrm{AVSD})$ and necrotizing enterocolitis $(n=1, \mathrm{VSD})$. In four children, the cause of death was not documented in the questionnaires (three AVSD and one PDA). The four children without a CHD died as a result of asphyxia, respiratory distress and infection, respectively, and one cause of death was unknown.

\section{Discussion}

This is the first large-scall study in the Netherlands regarding CHDs in children with DS. We found a prevalence of CHDs of $43 \%$, which is concordant with the prevalence presented in the literature of $44-58 \%$ [3, 4, $7,8,14,15,17,20]$, AVSD (54\%) was the most commonly occurring CHD in children with DS in our study. In our study, early diagnostic echocardiography was performed. Secundum ASD and persistent foramen ovale (PFO) were excluded as a CHD because a small secundum ASD is sometimes difficult to differentiate from a persistent foramen ovale, and the study design did not include follow-up to provide sufficient clarity about ASD versus PFO $[9,16]$. This could have caused an underestimation of the prevalence of CHD in our study. The early diagnostic procedure was used to make an early assessment of cardiac anatomy and in case of a significant defect to perform early intervention and to prevent pulmonary arterial hypertension in defects with a left to right shunt.

The inclusion of various cardiac defects and the use of different terminology in studies with children with DS made it difficult to compare these studies with our results. For instance, Stoll et al. did not report ASD as a CHD as well, which may explain why PDA is one of the three most prevalent defects in their study [7, 10, 14, 17]. In our study, there was no significant difference in the distribution of CHD according to ethnicity, as the subgroups were too small.

Freeman et al. reported in their study in the United States of America that black infants with DS had an AVSD more frequently than whites and that Hispanic infants with DS had an AVSD less frequently than whites [4]. In Chinese children with DS and a CHD, VSD was seen in $38 \%$ and AVSD in $25 \%$ [7]. AVSDs accounted for only $9 \%$ of the CHDs in Mexican children with DS, while ASD, VSD, and
PDA were most common. This study however began at the age 13 , so the mortality prior to the age of 13 may explain their distribution of CHDs [3].

Another relevant phenomenon which, to our knowledge, had not previously been appreciated and as such was not included in DS guidelines [2, 6] was the significantly increased incidence of PPHN in neonates with DS (5.2\%) we found in our study. This corresponds with Cua et al. who estimated the incidence of idiopathic PPHN in neonates with DS between $1.2 \%$ and $6.6 \%$, which is much higher than the reported overall incidence of $0.1 \%$ in the general population [1]. In our study, only seven children (28\%) had an AVSD, and in these children, we cannot exclude a relationship with the development of PPHN and nine (36\%) children with DS and PPHN did not have a CHD. In addition Shah et al. found no serious structural heart defect in $70 \%$ of children with DS and PPHN [13]. PPHN should be distinguished from another type of pulmonary hypertension, originating from a cardiac defect that permits left-to-right shunting and requires some period of time to be clinically detected. The newborn children with DS, who develop PPHN, are by contrast symptomatic just after birth [1, 13, 18].

Wren et al. found no clinical signs in the first weeks in some children with DS, with major cardiac malformations and associated pulmonary hypertension, even in some who progressed to irreversible pulmonary vascular disease [21]. A normal neonatal examination in children with DS does not therefore exclude a serious CHD [11]. This corresponds with our findings and emphasizes the importance of an early echocardiography of neonates with DS to detect any CHD and to determine the possible risk of developing pulmonary hypertension, as supported by the DS health care guidelines published by the American Academy of Pediatrics and the Down Syndrome Medical Interest Group of the United Kingdom and Ireland $[2,6]$. Because of the high incidence of a significant CHD in children with Down syndrome, the early recognition of CHD can lead to the optimal management of the defect. The surgical correction of significant defects usually takes place at the age of 2-4 months, though this happens earlier in certain cases (e.g. TOF) $[9,10]$.

In our study, basic neonatal characteristics, Apgar scores, birth weight and gestational age were not different in children with DS with and without CHD. This is in concords with Frid et al. who analysed the influence of CHD on these neonatal issues in 304 neonates with DS and also found no difference between those with and without CHD [5].

In the past decade, research has shown that neonatal mortality in DS appears to be becoming less dependent on CHDs and more often caused by neonatal pathology such as asphyxia, low birth weight and prematurity, as in the general population $[5,19]$. With regard to mortality, we should emphasize that with the exception of one child who died following a cardiac operation, the presence of a CHD 
was not responsible for reported mortality in children with DS in our recent study.

Our study has several limitations. Because of its retrospective design, we used data from questionnaires completed by paediatricians and have no on follow-up data. Furthermore, there was a sample bias since we could not use the data of all 820 children with DS who were registered by the DPSU, 630 (76.8\%) questionnaires were returned and not all questionnaires relating to children with trisomy 21 had complete data on cardiac status. On the other hand, the fall out cases were distributed between both the CHD and non-CHD children with DS.

Though the definition of PPHN was not specifically defined, diagnosis was based on the clinical assessment and an echocardiogram performed by the neonatologists and pediatric cardiologists who answered the questionnaires. It was impossible to draw a definitive conclusion on the exact role of the AVSD in the seven children with an AVSD in relation to the cause of the pulmonary hypertension; nevertheless, there were at least 18 other children (3.7\% of total) with PPHN that was not related to a CHD, which is still a high level.

In conclusion, we have demonstrated a $43 \%$ prevalence of CHD in neonates with DS and a significantly increased and elevated incidence of PPHN in neonates with DS (5.2\%) compared to the general population. Early recognizing the cardiac condition of neonates with DS seems justified. CHDs had no relationship with neonatal factors such as gestational age, Apgar score and birth weight of children with DS. There are no conflicts of interests

There are no conflicts of interests.

Open Access This article is distributed under the terms of the Creative Commons Attribution Noncommercial License which permits any noncommercial use, distribution, and reproduction in any medium, provided the original author(s) and source are credited.

\section{References}

1. Cua CL, Blankenship A, North AL et al (2007) Increased incidence of idiopathic persistent pulmonary hypertension in Down syndrome neonates. Pediatr Cardiol 28:250-254

2. Cunnif C, Frias JL, Kaye C et al (2001) Health supervision for children with Down syndrome, American Academy of Pediatrics. Pediatrics 107:442-449

3. De Rubens FJ, del Pozzo MB, Hach JLP et al (2003) Heart malformations in children with Down syndrome. Rev Esp Cardiol $56: 894-899$
4. Freeman SB, Bean LH, Allen EG et al (2008) Ethnicity, sex, and the incidence of congenital heart defects: a report from the National Down Syndrome Project. Genet Med 10:173-180

5. Frid C, Drott P, Otterblad OP et al (2004) Maternal and neonatal factors and mortality in children with Down syndrome born in 1973-1980 and 1995-1998. Acta Paediatr 93:106-112

6. Guidelines for basic essential medical surveillance. The Down's syndrome medical interest group UK and Ireland. Available at http://www.dsmig.org.uk/publications/guidelines.html. Accessed 29 January 2010.

7. Jacobs EG, Leung MP, Karlberg J (2000) Distribution of symptomatic congenital heart disease in Hong Kong. Pediatr Cardiol 21:148-157

8. Jaiyesimi O, Baichoo V (2007) Cardiovascular malformations in Omani Arab children with Down's syndrome. Cardiol Young 17:166-171

9. Kortenhorst MSQ, Hazekamp MG, Rammeloo LAJ et al (2005) Complete atrioventricular septal defect in children with Down's syndrome: good results of surgical correction at younger and younger ages. Ned Tijdschr Geneeskd 149:589-593

10. Lange R, Guenther T, Busch R et al (2007) The presence of Down syndrome is not a risk factor in complete atrioventricular septal defect repair. J Thorac Cardiovasc Surg 134:304-310

11. McElhinney DB, Straka M, Goldmuntz E et al (2002) Correlation between abnormal cardiac physical examination and echocardiographic findings in neonates with Down syndrome. Am J Med Genet 113:238-241

12. National Institute for Public Health and Environment, Rijks Instituut voor Volksgezondheid en Milieu (RIVM), National Dutch data collection on Health and Environment. Available at http://www.rivm.nl/vtv/object document/o1340n118479.html. Accessed 29 January 2010

13. Shah PS, Hellmann J, Adatia I (2004) Clinical characteristics and follow up of Down syndrome infants without congenital heart disease who presented with persistent pulmonary hypertension of newborn. J Perinat Med 32:168-170

14. Stoll C, Alembik Y, Dott B et al (1998) Study of Down syndrome in 238, 942 consecutive births. Ann Génét 41:44-51

15. Torfs CP, Christianson RE (1998) Anomalies in Down syndrome individuals in a large population-based registry. Am J Med Genet $77: 431-438$

16. Vis JC, Duffels MGJ, Winter MM et al (2009) Down syndrome: a cardiovascular perspective. J Intellect Disabil Res 53:419-425

17. Wahab AA, Bener A, Sandridge AL et al (2006) The pattern of Down syndrome among children in Qatar: a population-based study. Birth Defects Res A Clin Mol Teratol 76:609-612

18. Walsh-Sukys MC, Tyson JE, Wright LL et al (2000) Persistent pulmonary hypertension of the newborn in the era before nitric oxide: practice variation and outcomes. Pediatrics 105:14-20

19. Weijerman ME, van Furth AM, Vonk Noordegraaf A et al (2008) Prevalence, neonatal characteristics and first-year mortality of Down syndrome: a national study. J Pediatr 152:15-19

20. Wells GL, Barker SE, Finley SC et al (1994) Congenital heart disease in infants with Down's syndrome. South Med J 87:724 727

21. Wren C, Richmond S, Donaldson L (1999) Presentation of congenital heart disease in infancy: implications for routine examination. Arch Dis Child Fetal Neonatal Ed 80:F49-F53 